J. Korean Math. Soc. 49 (2012), No. 4, pp. 779-794

http://dx.doi.org/10.4134/JKMS.2012.49.4.779

\title{
GLOBAL STABILITY OF HIV INFECTION MODELS WITH INTRACELLULAR DELAYS
}

\author{
Ahmed Elaiw, Ismail Hassanien, and Shimaa Azoz
}

\begin{abstract}
In this paper, we study the global stability of two mathematical models for human immunodeficiency virus (HIV) infection with intracellular delays. The first model is a 5-dimensional nonlinear delay ODEs that describes the interaction of the HIV with two classes of target cells, $\mathrm{CD} 4^{+} \mathrm{T}$ cells and macrophages taking into account the saturation infection rate. The second model generalizes the first one by assuming that the infection rate is given by Beddington-DeAngelis functional response. Two time delays are used to describe the time periods between viral entry the two classes of target cells and the production of new virus particles. Lyapunov functionals are constructed and LaSalle-type theorem for delay differential equation is used to establish the global asymptotic stability of the uninfected and infected steady states of the HIV infection models. We have proven that if the basic reproduction number $R_{0}$ is less than unity, then the uninfected steady state is globally asymptotically stable and if the infected steady state exists, then it is globally asymptotically stable for all time delays.
\end{abstract}

\section{Introduction}

Modelling, analysis and control of human immunodeficiency virus (HIV) infection have attracted the interests of mathematicians during the recent years. Several mathematical models exist and adequately explain the interaction of the HIV infection and the immune system up to the stage of clinical latency, as well as viral suppression and immune system recovery after treatment therapy [18]. Some of these models are given by a system of nonlinear ODEs. These models are based on the assumption that, once the virus contacts a target cell, the cell begins producing new virus particles. However in real situation there is a lag between the time of viral entry a target cell and the time of producing new virus particles from the same target cell. Therefore, more accurate models have been proposed which are given by a system of nonlinear delay ODEs to account the intracellular time delay. The first HIV infection model accounting

Received April 29, 2011.

2010 Mathematics Subject Classification. 34D23, 92D25, 37B25, 92B05.

Key words and phrases. global stability, HIV dynamics, time delay, direct Lyapunov method. 
the intracellular time delay which represents the time between viral entry into a target cell and the production of new virus particles was proposed in 1996 by Herz et al. [7]. Thereafter, various models using discrete or distributed delays to model the intracellular phase were developed (see e.g. [2], [9], [10], [11], [12], [13], [14], [16], [17], [24], [27]). The basic HIV infection model with intracellular delay can be given in a general form as:

$$
\begin{aligned}
\dot{x}(t) & =f\left(x(t), x_{1}(t)\right)-g(x(t), v(t)), \\
\dot{x}_{1}(t) & =e^{-m \tau} g(x(t-\tau), v(t-\tau))-a x_{1}(t), \\
\dot{v}(t) & =p x_{1}(t)-r v(t),
\end{aligned}
$$

where $x(t), x_{1}(t)$ and $v(t)$, represent the concentrations of the uninfected CD $4^{+}$ $\mathrm{T}$ cells, infected $\mathrm{CD} 4^{+} \mathrm{T}$ cells and free virus particles, respectively. The function $f\left(x, x_{1}\right)$ represents the growth rate of the uninfected $\mathrm{CD} 4{ }^{+} \mathrm{T}$ cells and has been used in the literature in delayed HIV infection models in different forms:

- Growth rate without proliferation ([9], [11], [12], [14], [16], [17], [24], [27]):

$$
f\left(x, x_{1}\right)=\lambda-d x .
$$

- Growth rate with simple proliferation ([21], [23], [25]):

$$
f\left(x, x_{1}\right)=\lambda-d x+\alpha\left(1-\frac{x}{x_{\max }}\right) .
$$

- Growth rate with full proliferation ([2], [8]):

$$
f\left(x, x_{1}\right)=\lambda-d x+\alpha\left(1-\frac{x+x_{1}}{x_{\max }}\right) .
$$

The function $g(x, v)$ represents the incidence rate infection and it has been considered in the delayed HIV infection models by different forms:

- Bilinear incidence rate ([7], [8], [12], [14], [16]):

$$
g(x, v)=\beta x v .
$$

- Saturated incidence rate ([11], [23], [27]):

$$
g(x, v)=\frac{\beta x v}{1+b_{1} v} .
$$

- Beddington-DeAngelis infection rate ([9], [14], [24]):

$$
g(x, v)=\frac{\beta x v}{1+a_{1} x+b_{1} v} .
$$

Parameters $\lambda, d, \alpha, x_{\max }, \beta, a, p, r, a_{1}$ and $b_{1}$ are positive constants. Here, $\lambda$ represents the rate of which new $\mathrm{CD}^{+} \mathrm{T}$ cells are generated from sources within the body, $d$ is the death rate constant, $\alpha$ is the maximum proliferation rate of $\mathrm{CD}^{+} \mathrm{T}$ cells, $x_{\max }$ is maximum level of $\mathrm{CD} 4^{+} \mathrm{T}$ cells concentration in the body, and $\beta$ is the rate constant characterizing infection of the cells. Eq.(2) describes the population dynamics of the infected CD4 ${ }^{+} \mathrm{T}$ cells and shows that they die with rate constant $a$. The virus particles are produced by 
the infected $\mathrm{CD} 4^{+} \mathrm{T}$ cells with rate constant $p$, and are cleared from plasma with rate constant $r$. The parameter $\tau$ accounts for the time between viral entry into the $\mathrm{CD} 4^{+} \mathrm{T}$ cell and the production of new virus particles. The recruitment of virus producing cells at time $t$ is given by the number of cells that were newly infected $\mathrm{CD} 4{ }^{+} \mathrm{T}$ cells at time $t-\tau$ and are still alive at time $t$. If we assume a constant death rate $m$ for infected $\mathrm{CD} 4^{+} \mathrm{T}$ cells but not yet virus-producing cells, the probability of surviving the time period from $t-\tau$ to $t$ is $e^{-m \tau}$.

One extension of the basic delayed model (1)-(3) has been introduced by taking into account the Cytotoxic T Lymphocytes (CTL) immune response [15], [21], [22] and [26]. The role of CTL cells is to attack the infected cells. Another extension includes the addition of antiretroviral drug therapies [28] and [25]. A great effort has been devoted to study the basic and global properties of the HIV infection models with delay such as positive invariance properties, boundedness of the model solutions and stability analysis which are important for understanding the associated characteristics of the HIV dynamics (see e.g. [9], [11], [12], [14], [15], [23] and [27]).

All of the above mentioned delayed HIV infection models are mainly modelled the interaction of the HIV with one target cells, $\mathrm{CD} 4^{+} \mathrm{T}$ cells. Perleson et al., observed that after the rapid first phase of decay during the initial 1-2 weeks of antiretroviral treatment, plasma virus levels declined at a considerably slower rate [19]. This second phase of viral decay was attributed to the turnover of a longer-lived virus reservoir of infected cells. These cells are called macrophages and considered as the second target cell for the HIV. Therefore, the two target cells model is more accurate than the one target cells model (see [1] and [20]). Some HIV infection models exist to describe the interaction process of the HIV not only with the $\mathrm{CD} 4^{+} \mathrm{T}$ cells but also with the macrophages which are the crucial immune responses and play important roles in phagocytosis (see e.g. [1] and [20]). In very recent works ([3], [4] and [5]), we have proposed several HIV infection models with two target cells and investigated the global asymptotic stability of their steady states. However the intracellular time delay is neglected in these papers.

The purpose of the present paper is to study the global stability of two HIV infection models with two classes of target cells and delays. The first model is a 5-dimensional nonlinear delayed ODEs that describes the interaction of the HIV with two target cells, CD ${ }^{+} \mathrm{T}$ cells and macrophages taking into account the saturation infection rate. In the second model, the incidence rate is given by Beddington-DeAngelis functional response. The global stability of these models is established using Lyapunov functionals, which are similar in nature to those used in [10] and [27]. By constructing explicit Lyapunov functionals, we prove that the global dynamics of these models are determined by the basic reproduction number $R_{0}$. If $R_{0} \leq 1$, then the uninfected steady state is globally asymptotically stable (GAS). If the infected steady state exists, then it is GAS for all time delays. 


\subsection{HIV infection model with saturation infection rate}

We shall use the mathematical model of HIV infection proposed by ([1] and $[20]$ ), incorporating to take into account the intracellular delays and saturation infection rate. This model describes two co-circulation populations of target cells, potentially representing $\mathrm{CD} 4^{+} \mathrm{T}$ cells and macrophages and given by:

$$
\begin{aligned}
\dot{x}(t) & =\lambda_{1}-d_{1} x(t)-\frac{\beta_{1} x(t) v(t)}{1+v(t)}, \\
\dot{x}_{1}(t) & =e^{-m_{1} \tau_{1}} \frac{\beta_{1} x\left(t-\tau_{1}\right) v\left(t-\tau_{1}\right)}{1+v\left(t-\tau_{1}\right)}-a x_{1}(t), \\
\dot{y}(t) & =\lambda_{2}-d_{2} y(t)-\frac{\beta_{2} y(t) v(t)}{1+v(t)}, \\
\dot{y}_{1}(t) & =e^{-m_{2} \tau_{2}} \frac{\beta_{2} y\left(t-\tau_{2}\right) v\left(t-\tau_{2}\right)}{1+v\left(t-\tau_{2}\right)}-\delta y_{1}(t), \\
\dot{v}(t) & =p_{1} x_{1}(t)+p_{2} y_{1}(t)-r v(t),
\end{aligned}
$$

where $y$ and $y_{1}$ are the concentrations of the uninfected and infected macrophages, respectively. The populations of the macrophages are described by Eq.(6), where $\lambda_{2}$ represents the rate of which new macrophages cells are generated from sources within the body, $d_{2}$ is the death rate constant, and $\beta_{2}$ is the infection rate constant. In Eq.(7), $\delta$ is the death rate constant of the infected macrophages. The virus particles are produced by the infected CD ${ }^{+} \mathrm{T}$ cells and infected macrophages with rate constants $p_{1}$ and $p_{2}$, respectively. Here parameters $\tau_{1}$ and $\tau_{2}$ account for the times between viral entry into CD4 ${ }^{+} \mathrm{T}$ and macrophages cells, respectively, and the production of new virus particles. Also, $m_{1}$ and $m_{2}$ are assumed to be the constant death rates for infected CD4 ${ }^{+}$ $\mathrm{T}$ and macrophages cells, respectively, but not yet virus-producing cells. Thus, the probability of surviving the time period from $t-\tau_{i}$ to $t$ is $e^{-m_{i} \tau_{i}}, i=1,2$. The other variables and parameters have the same biological meaning as given in model (1)-(3). All the parameters of the model are supposed to be positive.

\subsection{Initial conditions}

The initial conditions for system (4)-(8) take the form

$$
\begin{aligned}
& x(\theta)=\varphi_{1}(\theta), x_{1}(\theta)=\varphi_{2}(\theta), y(\theta)=\varphi_{3}(\theta), y_{1}(\theta)=\varphi_{4}(\theta), v(\theta)=\varphi_{5}(\theta), \\
& \varphi_{i}(\theta) \geq 0, \theta \in\left[-\max \left\{\tau_{1}, \tau_{2}\right\}, 0\right), \varphi_{i}(0)>0, i=1, \ldots, 5,
\end{aligned}
$$

where $\left(\varphi_{1}(\theta), \ldots, \varphi_{5}(\theta)\right) \in C\left(\left[-\max \left\{\tau_{1}, \tau_{2}\right\}, 0\right], \mathbb{R}_{+}^{5}\right), C$ is the Banach space of continuous functions mapping the interval $\left[-\max \left\{\tau_{1}, \tau_{2}\right\}, 0\right]$ into $\mathbb{R}_{+}^{5}$.

By the fundamental theory of functional differential equations [6], system (4)-(8) has a unique solution $\left(x(t), x_{1}(t), y(t), y_{1}(t), v(t)\right)$ satisfying the initial conditions $(9)$. 


\subsection{Positivity and boundedness}

It is easy to show that all solutions of system (4)-(8) with initial conditions (9) are defined on $[0, \infty)$ and remain positive for all $t \geq 0$ (see [9] and [12]).

Proposition 1. The solution of (4)-(8) with the initial conditions (9) is ultimately bounded.

Proof. Let $X(t)=e^{-m_{1} \tau_{1}} x\left(t-\tau_{1}\right)+x_{1}(t)$ and $Y(t)=e^{-m_{2} \tau_{2}} y\left(t-\tau_{2}\right)+y_{1}(t)$. Then

$$
\begin{aligned}
& \dot{X}(t) \leq \lambda_{1} e^{-m_{1} \tau_{1}}-\sigma_{1} X(t), \\
& \dot{Y}(t) \leq \lambda_{2} e^{-m_{2} \tau_{2}}-\sigma_{2} Y(t),
\end{aligned}
$$

where $\sigma_{1}=\min \left\{d_{1}, a\right\}$ and $\sigma_{2}=\min \left\{d_{2}, \delta\right\}$. Hence $\lim \sup _{t \rightarrow \infty} X(t) \leq L_{1}$, and $\lim \sup _{t \rightarrow \infty} Y(t) \leq L_{2}$, where $L_{1}=\frac{\lambda_{1} e^{-m_{1} \tau_{1}}}{\sigma_{1}}$ and $L_{2}=\frac{\lambda_{2} e^{-m_{2} \tau_{2}}}{\sigma_{2}}$. On the other hand,

$$
\dot{v}(t) \leq p_{1} L_{1}+p_{2} L_{2}-r v,
$$

then $\limsup _{t \rightarrow \infty} v(t) \leq L_{3}$, where $L_{3}=\frac{p_{1} L_{1}+p_{2} L_{2}}{r}$. It follows that the solution of (4)-(8) is ultimately bounded.

\subsection{Steady states}

The dynamics of system (4)-(8) crucially depends on the basic reproduction number $R_{0}$ given by

$$
R_{0}=\frac{e^{-m_{1} \tau_{1}} p_{1} \beta_{1} \delta x_{0}+e^{-m_{2} \tau_{2}} p_{2} \beta_{2} a y_{0}}{a \delta r},
$$

where $x_{0}=\frac{\lambda_{1}}{d_{1}}, y_{0}=\frac{\lambda_{2}}{d_{2}}$. We note that $R_{0}$ can be written as:

$$
R_{0}=R_{1}+R_{2}
$$

where

$$
R_{1}=\frac{e^{-m_{1} \tau_{1}} p_{1} \beta_{1} x_{0}}{a r}, \quad R_{2}=\frac{e^{-m_{2} \tau_{2}} p_{2} \beta_{2} y_{0}}{\delta r},
$$

are the basic reproduction numbers of each T-cell and macrophages dynamics separately (see [3], [5]).

It is clear that, system (4)-(8) has an uninfected steady state $E_{0}=\left(x_{0}, 0, y_{0}\right.$, $0,0)$. The system can also has a positive infected steady state $E_{1}\left(x^{*}, x_{1}^{*}, y^{*}, y_{1}^{*}\right.$, $\left.v^{*}\right)$. The coordinates of the infected steady state, if they exist, satisfy the equalities:

$$
\begin{gathered}
\lambda_{1}=d_{1} x^{*}+\frac{\beta_{1} x^{*} v^{*}}{1+v^{*}}, \quad \lambda_{2}=d_{2} y^{*}+\frac{\beta_{2} y^{*} v^{*}}{1+v^{*}}, \\
a x_{1}^{*} e^{m_{1} \tau_{1}}=\frac{\beta_{1} x^{*} v^{*}}{1+v^{*}}, \quad \delta y_{1}^{*} e^{m_{2} \tau_{2}}=\frac{\beta_{2} y^{*} v^{*}}{1+v^{*}}, \quad r v^{*}=p_{1} x_{1}^{*}+p_{2} y_{1}^{*} .
\end{gathered}
$$




\subsection{Global stability analysis}

In this section, we shall consider the global stability of the uninfected and infected steady states of (4)-(8) by the Lyapunov direct method. To simplify the presentation we shall use the following notation: $z=z(t), z_{\tau_{i}}=z\left(t-\tau_{i}\right)$, $i=1,2$, for any $z \in\left\{x, x_{1}, y, y_{1}, v\right\}$. We also define a function $F: \mathbb{R}_{>0} \rightarrow \mathbb{R}_{\geq 0}$ as

$$
F(z)=z-1-\ln z
$$

We note that $F(z) \geq 0$ for any $z>0$ and has the global minimum $F(1)=0$.

Theorem 1. (i) If $R_{0} \leq 1$, then $E_{0}$ is GAS for any $\tau_{1}, \tau_{2} \geq 0$.

(ii) If $E_{1}$ exists, then it is GAS for any $\tau_{1}, \tau_{2} \geq 0$.

Proof. (i) We consider a Lyapunov functional

$$
\begin{aligned}
W_{1}= & x_{0} F\left(\frac{x}{x_{0}}\right)+e^{m_{1} \tau_{1}} x_{1}+\gamma\left[y_{0} F\left(\frac{y}{y_{0}}\right)+e^{m_{2} \tau_{2}} y_{1}\right]+\frac{a}{p_{1}} e^{m_{1} \tau_{1}} v \\
& +\int_{0}^{\tau_{1}} \frac{\beta_{1} x(t-\theta) v(t-\theta)}{1+v(t-\theta)} d \theta+\gamma \int_{0}^{\tau_{2}} \frac{\beta_{2} y(t-\theta) v(t-\theta)}{1+v(t-\theta)} d \theta,
\end{aligned}
$$

where $\gamma=\frac{p_{2} a}{p_{1} \delta} \frac{e^{m_{1} \tau_{1}}}{e^{m_{2} \tau_{2}}}$. We note that $W_{1}$ is defined, continuous and positive definite for all $\left(x, x_{1}, y, y_{1}, v\right)>0$ and $\theta \in\left[0, \max \left\{\tau_{1}, \tau_{2}\right\}\right]$. Also, the global minimum $W_{1}=0$ occurs at the uninfected steady state $E_{0}$. Further, function $W_{1}$ along the trajectories of (4)-(8) satisfies

$$
\begin{aligned}
\frac{d W_{1}}{d t}= & \left(1-\frac{x_{0}}{x}\right)\left(\lambda_{1}-d_{1} x-\frac{\beta_{1} x v}{1+v}\right)+e^{m_{1} \tau_{1}}\left(e^{-m_{1} \tau_{1}} \frac{\beta_{1} x_{\tau_{1}} v_{\tau_{1}}}{1+v_{\tau_{1}}}-a x_{1}\right) \\
& +\gamma\left[\left(1-\frac{y_{0}}{y}\right)\left(\lambda_{2}-d_{2} y-\frac{\beta_{2} y v}{1+v}\right)+e^{m_{2} \tau_{2}}\left(e^{-m_{2} \tau_{2}} \frac{\beta_{2} y_{\tau_{2}} v_{\tau_{2}}}{1+v_{\tau_{2}}}-\delta y_{1}\right)\right] \\
& +\frac{a}{p_{1}} e^{m_{1} \tau_{1}}\left(p_{1} x_{1}+p_{2} y_{1}-r v\right)+\frac{\beta_{1} x v}{1+v}-\frac{\beta_{1} x_{\tau_{1}} v_{\tau_{1}}}{1+v_{\tau_{1}}} \\
& +\gamma \frac{\beta_{2} y v}{1+v}-\gamma \frac{\beta_{2} y_{\tau_{2}} v_{\tau_{2}}}{1+v_{\tau_{2}}} \\
= & \lambda_{1}-d_{1} x-\frac{x_{0}}{x}\left(\lambda_{1}-d_{1} x-\frac{\beta_{1} x v}{1+v}\right) \\
& +\gamma\left[\lambda_{2}-d_{2} y-\frac{y_{0}}{y}\left(\lambda_{2}-d_{2} y-\frac{\beta_{2} y v}{1+v}\right)\right]-\frac{a r}{p_{1}} e^{m_{1} \tau_{1}} v \\
= & \lambda_{1}\left[2-\frac{x_{0}}{x}-\frac{x}{x_{0}}\right]+\gamma \lambda_{2}\left[2-\frac{y_{0}}{y}-\frac{y}{y_{0}}\right]+\frac{\beta_{1} x_{0} v}{1+v}+\gamma \frac{\beta_{2} y_{0} v}{1+v}-\frac{a r}{p_{1}} e^{m_{1} \tau_{1}} v \\
= & \lambda_{1}\left[2-\frac{x_{0}}{x}-\frac{x}{x_{0}}\right]+\gamma \lambda_{2}\left[2-\frac{y_{0}}{y}-\frac{y}{y_{0}}\right] \\
& +\frac{a r e^{m_{1} \tau_{1}} v}{p_{1}(1+v)}\left(\frac{e^{-m_{1} \tau_{1}} p_{1} \beta_{1} x_{0}}{a r}+\frac{e^{-m_{2} \tau_{2}} p_{2} \beta_{2} y_{0}}{\delta r}-1\right)-\frac{a r e^{m_{1} \tau_{1}} v^{2}}{p_{1}(1+v)}
\end{aligned}
$$




$$
\begin{aligned}
= & \lambda_{1}\left[2-\frac{x_{0}}{x}-\frac{x}{x_{0}}\right]+\gamma \lambda_{2}\left[2-\frac{y_{0}}{y}-\frac{y}{y_{0}}\right] \\
& +\frac{\operatorname{are}^{m_{1} \tau_{1}} v}{p_{1}(1+v)}\left(R_{0}-1\right)-\frac{\operatorname{are}^{m_{1} \tau_{1}} v^{2}}{p_{1}(1+v)} .
\end{aligned}
$$

Since the arithmetical mean is greater than or equal to the geometrical mean, then the first two terms of (11) are less than or equal to zero. Therefore, if $R_{0} \leq 1$, then $\frac{d W_{1}}{d t} \leq 0$ for all $x, x_{1}, y, y_{1}, v>0$. By Theorem 5.3.1 in [6], the solutions of system (4)-(8) limit to $M$, the largest invariant subset of $\left\{\frac{d W_{1}}{d t}=0\right\}$. Clearly, it follows from (11) that $\frac{d W_{1}}{d t}=0$ if and only if $x=x_{0}$, $y=y_{0}, v=0$. Each element of $M$ satisfies $x=x_{0}, y=y_{0}, v=0$ for all $t$, then $\dot{v}=0$. From Eq.(8) we drive that

$$
0=\dot{v}=p_{1} x_{1}+p_{2} y_{1}
$$

Since $x_{1}, y_{1} \geq 0$, then $p_{1} x_{1}+p_{2} y_{1}=0$ if and only if $x_{1}=y_{1}=0$. Hence $\frac{d W_{1}}{d t}=0$ if and only if $x=x_{0}, y=y_{0}, x_{1}=y_{1}=v=0$. From LaSalle's Invariance Principle, $E_{0}$ is GAS for any $\tau_{1}, \tau_{2} \geq 0$.

(ii) Define a Lyapunov functional

$$
\begin{aligned}
W_{2}= & x^{*} F\left(\frac{x}{x^{*}}\right)+e^{m_{1} \tau_{1}} x_{1}^{*} F\left(\frac{x_{1}}{x_{1}^{*}}\right)+\gamma\left(y^{*} F\left(\frac{y}{y^{*}}\right)+e^{m_{2} \tau_{2}} y_{1}^{*} F\left(\frac{y_{1}}{y_{1}^{*}}\right)\right) \\
& +\frac{a}{p_{1}} e^{m_{1} \tau_{1}} v^{*} F\left(\frac{v}{v^{*}}\right)+\frac{\beta_{1} x^{*} v^{*}}{1+v^{*}} \int_{0}^{\tau_{1}} F\left(\frac{x(t-\theta) v(t-\theta)\left(1+v^{*}\right)}{x^{*} v^{*}(1+v(t-\theta))}\right) d \theta \\
& +\gamma \frac{\beta_{2} y^{*} v^{*}}{1+v^{*}} \int_{0}^{\tau_{2}} F\left(\frac{y(t-\theta) v(t-\theta)\left(1+v^{*}\right)}{y^{*} v^{*}(1+v(t-\theta))}\right) d \theta .
\end{aligned}
$$

It is easy to see that $W_{2} \geq 0$ and $W_{2}=0$ if and only if $\left(x, x_{1}, y, y_{1}, v\right)$ take the steady state value $\left(x^{*}, x_{1}^{*}, y^{*}, y_{1}^{*}, v^{*}\right)$ and $x(t-\theta)=x^{*}, y(t-\theta)=y^{*}$, $v(t-\theta)=v^{*}$ for all $\theta \in\left[0, \max \left\{\tau_{1}, \tau_{2}\right\}\right]$.

Differentiating with respect to time yields

$$
\begin{aligned}
& \frac{d W_{2}}{d t} \\
= & \left(1-\frac{x^{*}}{x}\right)\left(\lambda_{1}-d_{1} x-\frac{\beta_{1} x v}{1+v}\right)+e^{m_{1} \tau_{1}}\left(1-\frac{x_{1}^{*}}{x_{1}}\right)\left(e^{-m_{1} \tau_{1}} \frac{\beta_{1} x_{\tau_{1}} v_{\tau_{1}}}{1+v_{\tau_{1}}}-a x_{1}\right) \\
& +\gamma\left[\left(1-\frac{y^{*}}{y}\right)\left(\lambda_{2}-d_{2} y-\frac{\beta_{2} y v}{1+v}\right)+e^{m_{2} \tau_{2}}\left(1-\frac{y_{1}^{*}}{y_{1}}\right)\left(e^{-m_{2} \tau_{2}} \frac{\beta_{2} y_{\tau_{2}} v_{\tau_{2}}}{1+v_{\tau_{2}}}-\delta y_{1}\right)\right] \\
& +\frac{a}{p_{1}} e^{m_{1} \tau_{1}}\left(1-\frac{v^{*}}{v}\right)\left(p_{1} x_{1}+p_{2} y_{1}-r v\right)+\frac{\beta_{1} x v}{1+v}-\frac{\beta_{1} x_{\tau_{1}} v_{\tau_{1}}}{1+v_{\tau_{1}}} \\
& +\frac{\beta_{1} x^{*} v^{*}}{1+v^{*}} \ln \left(\frac{x_{\tau_{1}} v_{\tau_{1}}(1+v)}{x v\left(1+v_{\tau_{1}}\right)}\right)
\end{aligned}
$$




$$
+\gamma\left[\frac{\beta_{2} y v}{1+v}-\frac{\beta_{2} y_{\tau_{2}} v_{\tau_{2}}}{1+v_{\tau_{2}}}+\frac{\beta_{2} y^{*} v^{*}}{1+v^{*}} \ln \left(\frac{y_{\tau_{2}} v_{\tau_{2}}(1+v)}{y v\left(1+v_{\tau_{2}}\right)}\right)\right] .
$$

Using the infected steady state conditions (10) and the following equality

$$
\begin{aligned}
\frac{a r}{p_{1}} e^{m_{1} \tau_{1}} v & =\frac{a r}{p_{1}} e^{m_{1} \tau_{1}} v^{*} \frac{v}{v^{*}} \\
& =\frac{a}{p_{1}} e^{m_{1} \tau_{1}}\left(p_{1} x_{1}^{*}+p_{2} y_{1}^{*}\right) \frac{v}{v^{*}} \\
& =a x_{1}^{*} e^{m_{1} \tau_{1}} \frac{v}{v^{*}}+\gamma \delta y_{1}^{*} e^{m_{2} \tau_{2}} \frac{v}{v^{*}},
\end{aligned}
$$

we obtain

$$
\begin{aligned}
\frac{d W_{2}}{d t}= & d_{1} x^{*}-d_{1} x-\frac{x^{*}}{x}\left(d_{1} x^{*}+a x_{1}^{*} e^{m_{1} \tau_{1}}-d_{1} x\right)+\frac{\beta_{1} x^{*} v}{1+v}-\frac{x_{1}^{*}}{x_{1}} \frac{\beta_{1} x_{\tau_{1}} v_{\tau_{1}}}{1+v_{\tau_{1}}} \\
& +3 a x_{1}^{*} e^{m_{1} \tau_{1}}-a x_{1}^{*} e^{m_{1} \tau_{1}} \frac{v}{v^{*}}-a x_{1}^{*} e^{m_{1} \tau_{1}} \frac{v^{*} x_{1}}{v x_{1}^{*}} \\
& +\gamma\left[d_{2} y^{*}-d_{2} y-\frac{y^{*}}{y}\left(d_{2} y^{*}+\delta y_{1}^{*} e^{m_{2} \tau_{2}}-d_{2} y\right)\right] \\
& +\gamma\left[\frac{\beta_{2} y^{*} v}{1+v}-\frac{y_{1}^{*}}{y_{1}} \frac{\beta_{2} y_{\tau_{2}} v_{\tau_{2}}}{1+v_{\tau_{2}}}+3 \delta y_{1}^{*} e^{m_{2} \tau_{2}}-\delta y_{1}^{*} e^{m_{2} \tau_{2}} \frac{v}{v^{*}}-\delta y_{1}^{*} e^{m_{2} \tau_{2}} \frac{v^{*} y_{1}}{v y_{1}^{*}}\right] \\
& +a x_{1}^{*} e^{m_{1} \tau_{1}} \ln \left(\frac{x_{\tau_{1}} v_{\tau_{1}}(1+v)}{x v\left(1+v_{\tau_{1}}\right)}\right)+\gamma \delta y_{1}^{*} e^{m_{2} \tau_{2}} \ln \left(\frac{y_{\tau_{2}} v_{\tau_{2}}(1+v)}{y v\left(1+v_{\tau_{2}}\right)}\right) \\
= & d_{1} x^{*}\left(2-\frac{x}{x^{*}}-\frac{x^{*}}{x}\right)-a x_{1}^{*} e^{m_{1} \tau_{1}} \frac{x^{*}}{x}+a x_{1}^{*} e^{m_{1} \tau_{1}} \frac{v}{v^{*}} \frac{\left(1+v^{*}\right)}{(1+v)} \\
& -a x_{1}^{*} e^{m_{1} \tau_{1}} \frac{x_{1}^{*} x_{\tau_{1}} v_{\tau_{1}}\left(1+v^{*}\right)}{x_{1} x^{*} v^{*}\left(1+v_{\tau_{1}}\right)}+3 a x_{1}^{*} e^{m_{1} \tau_{1}}-a x_{1}^{*} e^{m_{1} \tau_{1}} \frac{v}{v^{*}} \\
& -a x_{1}^{*} e^{m_{1} \tau_{1}} \frac{v^{*} x_{1}}{v x_{1}^{*}}+a x_{1}^{*} e^{m_{1} \tau_{1}} \ln \left(\frac{x_{\tau_{1}}}{x v\left(1+v_{\tau_{1}}(1+v)\right.}\right) \\
+ & {\left[d_{2} y^{*}\left(2-\frac{y}{y^{*}}-\frac{y^{*}}{y}\right)-\delta y_{1}^{*} e^{m_{2} \tau_{2}} \frac{y^{*}}{y}+\delta y_{1}^{*} e^{m_{2} \tau_{2}} \frac{v}{v^{*}} \frac{\left(1+v^{*}\right)}{(1+v)}\right.} \\
& \quad \delta y_{1}^{*} e^{m_{2} \tau_{2}} \frac{y_{1}^{*} y_{\tau_{2}} v_{\tau_{2}}\left(1+v^{*}\right)}{y_{1} y^{*} v^{*}\left(1+v_{\tau_{2}}\right)}+3 \delta y_{1}^{*} e^{m_{2} \tau_{2}}-\delta y_{1}^{*} e^{m_{2} \tau_{2}} \frac{v}{v^{*}} \\
& \left.\quad \delta y_{1}^{*} e^{m_{2} \tau_{2}} \frac{v^{*} y_{1}}{v y_{1}^{*}}+\delta y_{1}^{*} e^{m_{2} \tau_{2}} \ln \left(\frac{y_{\tau_{2}} v_{\tau_{2}}(1+v)}{y v\left(1+v_{\tau_{2}}\right)}\right)\right]
\end{aligned}
$$

and using also the following equalities

$$
\begin{aligned}
\ln \left(\frac{x_{\tau_{1}} v_{\tau_{1}}(1+v)}{x v\left(1+v_{\tau_{1}}\right)}\right)= & \ln \left(\frac{x^{*}}{x}\right)+\ln \left(\frac{x_{1} v^{*}}{x_{1}^{*} v}\right)+\ln \left(\frac{x_{1}^{*} x_{\tau_{1}} v_{\tau_{1}}\left(1+v^{*}\right)}{x_{1} x^{*} v^{*}\left(1+v_{\tau_{1}}\right)}\right) \\
& +\ln \left(\frac{1+v}{1+v^{*}}\right), \\
\ln \left(\frac{y_{\tau_{2}} v_{\tau_{2}}(1+v)}{y v\left(1+v_{\tau_{2}}\right)}\right)= & \ln \left(\frac{y^{*}}{y}\right)+\ln \left(\frac{y_{1} v^{*}}{y_{1}^{*} v}\right)+\ln \left(\frac{y_{1}^{*} y_{\tau_{2}} v_{\tau_{2}}\left(1+v^{*}\right)}{y_{1} y^{*} v^{*}\left(1+v_{\tau_{2}}\right)}\right)
\end{aligned}
$$


we get

$$
\begin{gathered}
+\ln \left(\frac{1+v}{1+v^{*}}\right), \\
-1-\frac{v}{v^{*}}+\frac{v}{v^{*}} \frac{\left(1+v^{*}\right)}{(1+v)}+\frac{1+v}{1+v^{*}}=-\frac{\left(v-v^{*}\right)^{2}}{v^{*}(1+v)\left(1+v^{*}\right)},
\end{gathered}
$$

$$
\begin{aligned}
& \frac{d W_{2}}{d t}=d_{1} x^{*}\left(2-\frac{x}{x^{*}}-\frac{x^{*}}{x}\right)+\gamma d_{2} y^{*}\left(2-\frac{y}{y^{*}}-\frac{y^{*}}{y}\right) \\
&-a x_{1}^{*} e^{m_{1} \tau_{1}}\left[F\left(\frac{x^{*}}{x}\right)+F\left(\frac{x_{1} v^{*}}{x_{1}^{*} v}\right)+F\left(\frac{1+v}{1+v^{*}}\right)\right. \\
&\left.+F\left(\frac{x_{1}^{*} x_{\tau_{1}} v_{\tau_{1}}\left(1+v^{*}\right)}{x_{1} x^{*} v^{*}\left(1+v_{\tau_{1}}\right)}\right)+\frac{\left(v-v^{*}\right)^{2}}{v^{*}(1+v)\left(1+v^{*}\right)}\right] \\
&-\gamma \delta y_{1}^{*} e^{m_{2} \tau_{2}}[ {\left[F\left(\frac{y^{*}}{y}\right)+F\left(\frac{y_{1} v^{*}}{y_{1}^{*} v}\right)+F\left(\frac{1+v}{1+v^{*}}\right)\right.} \\
&\left.+F\left(\frac{y_{1}^{*} y_{\tau_{2}} v_{\tau_{2}}\left(1+v^{*}\right)}{y_{1} y^{*} v^{*}\left(1+v_{\tau_{2}}\right)}\right)+\frac{\left(v-v^{*}\right)^{2}}{v^{*}(1+v)\left(1+v^{*}\right)}\right] .
\end{aligned}
$$

Since the arithmetical mean is greater than or equal to the geometrical mean, then the first two terms of (12) are less than or equal to zero. It is easy to see that if $x^{*}, x_{1}^{*}, y^{*}, y_{1}^{*}, v^{*}>0$, then $\frac{d W_{2}}{d t} \leq 0$. By Theorem 5.3.1 in [6], the solutions of system (4)-(8) limit to $M$, the largest invariant subset of $\left\{\frac{d W_{2}}{d t}=0\right\}$. It can be seen that $\frac{d W_{2}}{d t}=0$ if and only if $x=x^{*}, y=y^{*}, v=v^{*}$, and $F=0$ i.e.,

$$
\frac{x_{1} v^{*}}{x_{1}^{*} v}=\frac{y_{1} v^{*}}{y_{1}^{*} v}=\frac{x_{1}^{*} x_{\tau_{1}} v_{\tau_{1}}\left(1+v^{*}\right)}{x_{1} x^{*} v^{*}\left(1+v_{\tau_{1}}\right)}=\frac{y_{1}^{*} y_{\tau_{2}} v_{\tau_{2}}\left(1+v^{*}\right)}{y_{1} y^{*} v^{*}\left(1+v_{\tau_{2}}\right)}=1 .
$$

If $v=v^{*}$, then from (13) we have $x_{1}=x_{1}^{*}$ and $y_{1}=y_{1}^{*}$. LaSalle's Invariance Principle implies global stability of $E_{1}$.

\section{HIV infection model with Beddington-DeAngelis functional response}

In this section we study the global stability of HIV infection model with two target cells and delays by assuming that the infection rate is given by the Beddington-DeAngelis functional response:

$$
\begin{aligned}
\dot{x}(t) & =\lambda_{1}-d_{1} x(t)-\frac{\beta_{1} x(t) v(t)}{1+a_{1} x(t)+b_{1} v(t)}, \\
\dot{x}_{1}(t) & =e^{-m_{1} \tau_{1}} \frac{\beta_{1} x\left(t-\tau_{1}\right) v\left(t-\tau_{1}\right)}{1+a_{1} x\left(t-\tau_{1}\right)+b_{1} v\left(t-\tau_{1}\right)}-a x_{1}(t), \\
\dot{y}(t) & =\lambda_{2}-d_{2} y(t)-\frac{\beta_{2} y(t) v(t)}{1+a_{2} y(t)+b_{2} v(t)}, \\
\dot{y}_{1}(t) & =e^{-m_{2} \tau_{2}} \frac{\beta_{2} y\left(t-\tau_{2}\right) v\left(t-\tau_{2}\right)}{1+a_{2} y\left(t-\tau_{2}\right)+b_{2} v\left(t-\tau_{2}\right)}-\delta y_{1}(t),
\end{aligned}
$$




$$
\dot{v}(t)=p_{1} x_{1}(t)+p_{2} y_{1}(t)-r v(t) .
$$

Here $\frac{\beta_{1} x v}{1+a_{1} x+b_{1} v}$ and $\frac{\beta_{2} y v}{1+a_{2} y+b_{2} v}$, represent the Beddington-DeAngelis functional response of the $\mathrm{CD} 4^{+} \mathrm{T}$ cells and macrophages, respectively, where $a_{1}, b_{1}, a_{2}, b_{2}$ are positive constants. All the variables and parameters of the model have the same definitions as given in the previous section. This model can be considered as an extension of the model given in [9], [14] which describes the interaction of the HIV with one target cells, $\mathrm{CD} 4^{+} \mathrm{T}$ cells.

\subsection{Steady states}

It is clear that, system (14)-(18) has an uninfected steady state $E_{0}=$ $\left(x_{0}, 0, y_{0}, 0,0\right)$, where $x_{0}=\frac{\lambda_{1}}{d_{1}}, y_{0}=\frac{\lambda_{2}}{d_{2}}$. The system can also has a positive infected steady state $E_{1}\left(x^{*}, x_{1}^{*}, y^{*}, y_{1}^{*}, v^{*}\right)$. The coordinates of the infected steady state, if they exist, satisfy the equalities:

$$
\begin{aligned}
\lambda_{1} & =d_{1} x^{*}+\frac{\beta_{1} x^{*} v^{*}}{1+a_{1} x^{*}+b_{1} v^{*}}, \\
\lambda_{2} & =d_{2} y^{*}+\frac{\beta_{2} y^{*} v^{*}}{1+a_{2} y^{*}+b_{2} v^{*}}, \\
a x_{1}^{*} e^{m_{1} \tau_{1}} & =\frac{\beta_{1} x^{*} v^{*}}{1+a_{1} x^{*}+b_{1} v^{*}}, \\
\delta y_{1}^{*} e^{m_{2} \tau_{2}} & =\frac{\beta_{2} y^{*} v^{*}}{1+a_{2} y^{*}+b_{2} v^{*}}, \\
r v^{*} & =p_{1} x_{1}^{*}+p_{2} y_{1}^{*} .
\end{aligned}
$$

The basic reproduction number $R_{0}$ for system (14)-(18) is given by:

$$
R_{0}=\frac{e^{-m_{1} \tau_{1}} p_{1} \beta_{1} x_{0} \delta\left(1+a_{2} y_{0}\right)+e^{-m_{2} \tau_{2}} p_{2} \beta_{2} y_{0} a\left(1+a_{1} x_{0}\right)}{a \delta r\left(1+a_{1} x_{0}\right)\left(1+a_{2} y_{0}\right)} .
$$

We note that $R_{0}$ can be written as:

$$
R_{0}=R_{1}+R_{2}
$$

where

$$
R_{1}=\frac{e^{-m_{1} \tau_{1}} p_{1} \beta_{1} x_{0}}{\operatorname{ar}\left(1+a_{1} x_{0}\right)}, \quad R_{2}=\frac{e^{-m_{2} \tau_{2}} p_{2} \beta_{2} y_{0}}{\delta r\left(1+a_{2} y_{0}\right)} .
$$

\subsection{Global stability analysis}

In this section, we prove the global stability of the uninfected and infected steady states of system (14)-(18).

Theorem 2. (i) If $R_{0} \leq 1$, then $E_{0}$ is GAS for any $\tau_{1}, \tau_{2} \geq 0$.

(ii) If $E_{1}$ exists, then it is GAS for any $\tau_{1}, \tau_{2} \geq 0$.

Proof. We consider a Lyapunov functional

$$
W_{1}=\frac{x_{0}}{1+a_{1} x_{0}} F\left(\frac{x}{x_{0}}\right)+e^{m_{1} \tau_{1}} x_{1}+\gamma \frac{y_{0}}{1+a_{2} y_{0}} F\left(\frac{y}{y_{0}}\right)+\gamma e^{m_{2} \tau_{2}} y_{1}
$$




$$
\begin{aligned}
& +\frac{a}{p_{1}} e^{m_{1} \tau_{1}} v+\int_{0}^{\tau_{1}} \frac{\beta_{1} x(t-\theta) v(t-\theta)}{1+a_{1} x(t-\theta)+b_{1} v(t-\theta)} d \theta \\
& +\gamma \int_{0}^{\tau_{2}} \frac{\beta_{2} y(t-\theta) v(t-\theta)}{1+a_{2} y(t-\theta)+b_{2} v(t-\theta)} d \theta .
\end{aligned}
$$

We note that $W_{1}$ is defined, continuous and positive definite for all $\left(x, x_{1}, y, y_{1}\right.$, $v)>0$. Also, the global minimum $W_{1}=0$ occurs at the uninfected steady state $E_{0}$. The time derivative of $W_{1}$ along the solution of (14)-(18) is given by

$$
\begin{aligned}
& \frac{d W_{1}}{d t}=\frac{1}{1+a_{1} x_{0}}\left(1-\frac{x_{0}}{x}\right)\left(\lambda_{1}-d_{1} x-\frac{\beta_{1} x v}{1+a_{1} x+b_{1} v}\right)+\frac{\beta_{1} x_{\tau_{1}} v_{\tau_{1}}}{1+a_{1} x_{\tau_{1}}+b_{1} v_{\tau_{1}}} \\
& -a e^{m_{1} \tau_{1}} x_{1}+\gamma\left[\frac{1}{\left(1+a_{2} y_{0}\right)}\left(1-\frac{y_{0}}{y}\right)\left(\lambda_{2}-d_{2} y-\frac{\beta_{2} y v}{1+a_{2} y+b_{2} v}\right)\right. \\
& \left.+\frac{\beta_{2} y_{\tau_{2}} v_{\tau_{2}}}{1+a_{2} y_{\tau_{2}}+b_{2} v_{\tau_{2}}}-\delta e^{m_{2} \tau_{2}} y_{1}\right]+\frac{a}{p_{1}} e^{m_{1} \tau_{1}}\left(p_{1} x_{1}+p_{2} y_{1}-r v\right) \\
& +\frac{\beta_{1} x v}{1+a_{1} x+b_{1} v}-\frac{\beta_{1} x_{\tau_{1}} v_{\tau_{1}}}{1+a_{1} x_{\tau_{1}}+b_{1} v_{\tau_{1}}}+\gamma \frac{\beta_{2} y v}{1+a_{2} y+b_{2} v} \\
& -\gamma \frac{\beta_{2} y_{\tau_{2}} v_{\tau_{2}}}{1+a_{2} y_{\tau_{2}}+b_{2} v_{\tau_{2}}} \\
& =\frac{\lambda_{1}}{1+a_{1} x_{0}}\left[2-\frac{x}{x_{0}}-\frac{x_{0}}{x}\right]+\gamma \frac{\lambda_{2}}{\left(1+a_{2} y_{0}\right)}\left[2-\frac{y}{y_{0}}-\frac{y_{0}}{y}\right] \\
& -\frac{1}{\left(1+a_{1} x_{0}\right)} \frac{\beta_{1} x v}{\left(1+a_{1} x+b_{1} v\right)}+\frac{1}{\left(1+a_{1} x_{0}\right)} \frac{\beta_{1} x_{0} v}{\left(1+a_{1} x+b_{1} v\right)} \\
& +\frac{\beta_{1} x v}{1+a_{1} x+b_{1} v}-\frac{a r}{2 p_{1}} e^{m_{1} \tau_{1}} v+\gamma\left[-\frac{1}{\left(1+a_{2} y_{0}\right)} \frac{\beta_{2} y v}{\left(1+a_{2} y+b_{2} v\right)}\right. \\
& \left.+\frac{1}{\left(1+a_{2} y_{0}\right)} \frac{\beta_{2} y_{0} v}{\left(1+a_{2} y+b_{2} v\right)}+\frac{\beta_{2} y v}{1+a_{2} y+b_{2} v}-\frac{\delta r}{2 p_{2}} e^{m_{2} \tau_{2}} v\right] \\
& =\frac{\lambda_{1}}{1+a_{1} x_{0}}\left[2-\frac{x}{x_{0}}-\frac{x_{0}}{x}\right]+\gamma \frac{\lambda_{2}}{\left(1+a_{2} y_{0}\right)}\left[2-\frac{y}{y_{0}}-\frac{y_{0}}{y}\right] \\
& +\frac{a r e^{m_{1} \tau_{1}} v\left(1+a_{1} x\right)}{p_{1}\left(1+a_{1} x+b_{1} v\right)}\left(R_{1}-\frac{1}{2}\right)-\frac{a r b_{1} e^{m_{1} \tau_{1}} v^{2}}{2 p_{1}\left(1+a_{1} x+b_{1} v\right)} \\
& +\frac{a r e^{m_{1} \tau_{1}} v\left(1+a_{2} y\right)}{p_{1}\left(1+a_{2} y+b_{2} v\right)}\left(R_{2}-\frac{1}{2}\right)-\frac{a r b_{2} e^{m_{1} \tau_{1}} v^{2}}{2 p_{1}\left(1+a_{2} y+b_{2} v\right)}, \\
& \frac{d W_{1}}{d t}=\frac{\lambda_{1}}{1+a_{1} x_{0}}\left[2-\frac{x}{x_{0}}-\frac{x_{0}}{x}\right]+\gamma \frac{\lambda_{2}}{\left(1+a_{2} y_{0}\right)}\left[2-\frac{y}{y_{0}}-\frac{y_{0}}{y}\right] \\
& +\frac{a r e^{m_{1} \tau_{1}} v\left(1+a_{1} x\right)\left(1+a_{2} y\right)}{p_{1}\left(1+a_{1} x+b_{1} v\right)\left(1+a_{2} y+b_{2} v\right)}\left(R_{0}-1\right) \\
& +\frac{a r b_{2} e^{m_{1} \tau_{1}} v^{2}\left(1+a_{1} x\right)}{p_{1}\left(1+a_{1} x+b_{1} v\right)\left(1+a_{2} y+b_{2} v\right)}\left(R_{1}-1\right)
\end{aligned}
$$




$$
\begin{aligned}
& +\frac{a r b_{1} e^{m_{1} \tau_{1}} v^{2}\left(1+a_{2} y\right)}{p_{1}\left(1+a_{1} x+b_{1} v\right)\left(1+a_{2} y+b_{2} v\right)}\left(R_{2}-1\right) \\
& -\frac{a r b_{1} b_{2} e^{m_{1} \tau_{1}} v^{3}}{p_{1}\left(1+a_{1} x+b_{1} v\right)\left(1+a_{2} y+b_{2} v\right)} .
\end{aligned}
$$

Since the arithmetical mean is greater than or equal to the geometrical mean, then the first two terms of (24) are less than or equal to zero. Therefore, if $R_{0} \leq 1$, then $R_{1}, R_{2} \leq 1$ and $\frac{d W_{1}}{d t} \leq 0$ for all $x, x_{1}, y, y_{1}, v>0$. The global stability of $E_{0}$ follows from LaSalle's Invariance Principle.

(ii) Define a Lyapunov functional

$$
\begin{aligned}
W_{2}= & x-x^{*}-\int_{x^{*}}^{x} \frac{x^{*}\left(1+a_{1} \eta+b_{1} v^{*}\right)}{\eta\left(1+a_{1} x^{*}+b_{1} v^{*}\right)} d \eta+e^{m_{1} \tau_{1}} x_{1}^{*} F\left(\frac{x_{1}}{x_{1}^{*}}\right) \\
& +\gamma\left(y-y^{*}-\int_{y^{*}}^{y} \frac{y^{*}\left(1+a_{2} \eta+b_{2} v^{*}\right)}{\eta\left(1+a_{2} y^{*}+b_{2} v^{*}\right)} d \eta+e^{m_{2} \tau_{2}} y_{1}^{*} F\left(\frac{y_{1}}{y_{1}^{*}}\right)\right) \\
& +\frac{a}{p_{1}} e^{m_{1} \tau_{1}} v^{*} F\left(\frac{v}{v^{*}}\right) \\
& +\frac{\beta_{1} x^{*} v^{*}}{1+a_{1} x^{*}+b_{1} v^{*}} \int_{0}^{\tau_{1}} F\left(\frac{x(t-\theta) v(t-\theta)\left(1+a_{1} x^{*}+b_{1} v^{*}\right)}{x^{*} v^{*}\left(1+a_{1} x(t-\theta)+b_{1} v(t-\theta)\right)}\right) d \theta \\
& +\gamma \frac{\beta_{2} y^{*} v^{*}}{1+a_{2} y^{*}+b_{2} v^{*}} \int_{0}^{\tau_{2}} F\left(\frac{y(t-\theta) v(t-\theta)\left(1+a_{2} y^{*}+b_{2} v^{*}\right)}{y^{*} v^{*}\left(1+a_{2} y(t-\theta)+b_{2} v(t-\theta)\right)}\right) d \theta .
\end{aligned}
$$

Differentiating with respect to time yields

$$
\begin{aligned}
\frac{d W_{2}}{d t}= & \left(1-\frac{x^{*}}{x} \frac{1+a_{1} x+b_{1} v^{*}}{1+a_{1} x^{*}+b_{1} v^{*}}\right)\left(\lambda_{1}-d_{1} x-\frac{\beta_{1} x v}{1+a_{1} x+b_{1} v}\right) \\
& +e^{m_{1} \tau_{1}}\left(1-\frac{x_{1}^{*}}{x_{1}}\right)\left(\frac{e^{-m_{1} \tau_{1}} \beta_{1} x_{\tau_{1}} v_{\tau_{1}}}{1+a_{1} x_{\tau_{1}}+b_{1} v_{\tau_{1}}}-a x_{1}\right) \\
& +\gamma\left[\left(1-\frac{y^{*}}{y} \frac{1+a_{2} y+b_{2} v^{*}}{1+a_{2} y^{*}+b_{2} v^{*}}\right)\left(\lambda_{2}-d_{2} y-\frac{\beta_{2} y v}{1+a_{2} y+b_{2} v}\right)\right. \\
& \left.+e^{m_{2} \tau_{2}}\left(1-\frac{y_{1}^{*}}{y_{1}}\right)\left(\frac{e^{-m_{2} \tau_{2}} \beta_{2} y_{\tau_{2}} v_{\tau_{2}}}{1+a_{2} y_{\tau_{2}}+b_{2} v_{\tau_{2}}}-\delta y_{1}\right)\right] \\
& +\frac{a}{p_{1}} e^{m_{1} \tau_{1}}\left(1-\frac{v^{*}}{v}\right)\left(p_{1} x_{1}+p_{2} y_{1}-r v\right)+\frac{\beta_{1} x v}{1+a_{1} x+b_{1} v} \\
& -\frac{\beta_{1} x_{\tau_{1}} v_{\tau_{1}}}{1+a_{1} x_{\tau_{1}}+b_{1} v_{\tau_{1}}}+\frac{\beta_{1} x^{*} v^{*}}{1+a_{1} x^{*}+b_{1} v^{*}} \ln \left(\frac{x_{\tau_{1}} v_{\tau_{1}}\left(1+a_{1} x+b_{1} v\right)}{x v\left(1+a_{1} x_{\tau_{1}}+b_{1} v_{\tau_{1}}\right)}\right) \\
& +\gamma \frac{\beta_{2} y v}{1+a_{2} y+b_{2} v}-\gamma \frac{\beta_{2} y_{\tau_{2}} v_{\tau_{2}}}{1+a_{2} y_{\tau_{2}}+b_{2} v_{\tau_{2}}} \\
& +\gamma \frac{\beta_{2} y^{*} v^{*}}{1+a_{2} y^{*}+b_{2} v^{*}} \ln \left(\frac{y_{\tau_{2}} v_{\tau_{2}}\left(1+a_{2} y+b_{2} v\right)}{y v\left(1+a_{2} y_{\tau_{2}}+b_{2} v_{\tau_{2}}\right)}\right) .
\end{aligned}
$$


Using the infected steady state $E_{1}$ conditions (19)-(23) we obtain

$$
\begin{aligned}
& \frac{d W_{2}}{d t}=\left(1-\frac{x^{*}}{x} \frac{1+a_{1} x+b_{1} v^{*}}{1+a_{1} x^{*}+b_{1} v^{*}}\right)\left(d_{1} x^{*}-d_{1} x\right) \\
& -a x_{1}^{*} e^{m_{1} \tau_{1}} \frac{x^{*}}{x} \frac{1+a_{1} x+b_{1} v^{*}}{1+a_{1} x^{*}+b_{1} v^{*}} \\
& +3 a x_{1}^{*} e^{m_{1} \tau_{1}}+a x_{1}^{*} e^{m_{1} \tau_{1}} \frac{v}{v^{*}} \frac{1+a_{1} x+b_{1} v^{*}}{1+a_{1} x+b_{1} v} \\
& -a x_{1}^{*} e^{m_{1} \tau_{1}} \frac{x_{1}^{*} x_{\tau_{1}} v_{\tau_{1}}\left(1+a_{1} x^{*}+b_{1} v^{*}\right)}{x_{1} x^{*} v^{*}\left(1+a_{1} x_{\tau_{1}}+b_{1} v_{\tau_{1}}\right)} \\
& -a x_{1}^{*} e^{m_{1} \tau_{1}} \frac{v}{v^{*}}-a x_{1}^{*} e^{m_{1} \tau_{1}} \frac{v^{*} x_{1}}{v x_{1}^{*}} \\
& +a x_{1}^{*} e^{m_{1} \tau_{1}} \ln \left(\frac{x_{\tau_{1}} v_{\tau_{1}}\left(1+a_{1} x+b_{1} v\right)}{x v\left(1+a_{1} x_{\tau_{1}}+b_{1} v_{\tau_{1}}\right)}\right) \\
& +\gamma\left[\left(1-\frac{y^{*}}{y} \frac{1+a_{2} y+b_{2} v^{*}}{1+a_{2} y^{*}+b_{2} v^{*}}\right)\left(d_{2} y^{*}-d_{2} y^{*}\right)\right. \\
& -\delta y_{1}^{*} e^{m_{2} \tau_{2}} \frac{y^{*}}{y} \frac{1+a_{2} y+b_{2} v^{*}}{1+a_{2} y^{*}+b_{2} v^{*}}+3 \delta y_{1}^{*} e^{m_{2} \tau_{2}} \\
& +\delta y_{1}^{*} e^{m_{2} \tau_{2}} \frac{v}{v^{*}} \frac{1+a_{2} y+b_{2} v^{*}}{1+a_{2} y+b_{2} v} \\
& -\delta y_{1}^{*} e^{m_{2} \tau_{2}} \frac{y_{1}^{*} y_{\tau_{2}} v_{\tau_{2}}\left(1+a_{2} y^{*}+b_{2} v^{*}\right)}{y_{1} y^{*} v^{*}\left(1+a_{2} y_{\tau_{2}}+b_{2} v_{\tau_{2}}\right)} \\
& -\delta y_{1}^{*} e^{m_{2} \tau_{2}} \frac{v}{v^{*}}-\delta y_{1}^{*} e^{m_{2} \tau_{2}} \frac{v^{*} y_{1}}{v y_{1}^{*}} \\
& \left.+\gamma \delta y_{1}^{*} e^{m_{2} \tau_{2}} \ln \left(\frac{y_{\tau_{2}} v_{\tau_{2}}\left(1+a_{2} y+b_{2} v\right)}{y v\left(1+a_{2} y_{\tau_{2}}+b_{2} v_{\tau_{2}}\right)}\right)\right] .
\end{aligned}
$$

By a straightforward calculations we get for $x$ :

$$
\begin{aligned}
\left(1-\frac{x^{*}}{x} \frac{1+a_{1} x+b_{1} v^{*}}{1+a_{1} x^{*}+b_{1} v^{*}}\right)\left(d_{1} x^{*}-d_{1} x\right)=-\frac{d_{1}\left(x-x^{*}\right)^{2}\left(1+b_{1} v^{*}\right)}{x\left(1+a_{1} x^{*}+b_{1} v^{*}\right)} \\
-1+\frac{v}{v^{*}} \frac{1+a_{1} x+b_{1} v^{*}}{1+a_{1} x+b_{1} v}-\frac{v}{v^{*}}+\frac{1+a_{1} x+b_{1} v}{1+a_{1} x+b_{1} v^{*}} \\
=-\frac{b_{1}\left(1+a_{1} x\right)\left(v-v^{*}\right)^{2}}{v^{*}\left(1+a_{1} x+b_{1} v\right)\left(1+a_{1} x+b_{1} v^{*}\right)} \\
\quad \ln \left(\frac{x_{\tau_{1}} v_{\tau_{1}}\left(1+a_{1} x+b_{1} v\right)}{x v\left(1+a_{1} x_{\tau_{1}}+b_{1} v_{\tau_{1}}\right)}\right) \\
=\ln \left(\frac{x^{*}}{x} \frac{1+a_{1} x+b_{1} v^{*}}{1+a_{1} x^{*}+b_{1} v^{*}}\right)+\ln \left(\frac{x_{1} v^{*}}{x_{1}^{*} v}\right)
\end{aligned}
$$




$$
+\ln \left(\frac{1+a_{1} x+b_{1} v}{1+a_{1} x+b_{1} v^{*}}\right)+\ln \left(\frac{x_{1}^{*} x_{\tau_{1}} v_{\tau_{1}}\left(1+a_{1} x^{*}+b_{1} v^{*}\right)}{x_{1} x^{*} v^{*}\left(1+a_{1} x_{\tau_{1}}+b_{1} v_{\tau_{1}}\right)}\right) .
$$

Similar equalities can be deduced for $y$. Then, $\frac{d W_{2}}{d t}$ can be written as:

$$
\begin{aligned}
\frac{d W_{2}}{d t}= & -\frac{d_{1}\left(x-x^{*}\right)^{2}\left(1+b_{1} v^{*}\right)}{x\left(1+a_{1} x^{*}+b_{1} v^{*}\right)} \\
& -a x_{1}^{*} e^{m_{1} \tau_{1}} \frac{b_{1}\left(1+a_{1} x\right)\left(v-v^{*}\right)^{2}}{v^{*}\left(1+a_{1} x+b_{1} v\right)\left(1+a_{1} x+b_{1} v^{*}\right)} \\
& -\frac{\gamma d_{2}\left(y-y^{*}\right)^{2}\left(1+b_{2} v^{*}\right)}{y\left(1+a_{2} y^{*}+b_{2} v^{*}\right)} \\
& -\delta y_{1}^{*} e^{m_{2} \tau_{2}} \frac{\gamma b_{2}\left(1+a_{2} y\right)\left(v-v^{*}\right)^{2}}{v^{*}\left(1+a_{2} y+b_{2} v\right)\left(1+a_{2} y+b_{2} v^{*}\right)} \\
& -a x_{1}^{*} e^{m_{1} \tau_{1}}\left[F\left(\frac{x^{*}}{x} \frac{1+a_{1} x+b_{1} v^{*}}{1+a_{1} x^{*}+b_{1} v^{*}}\right)+F\left(\frac{x_{1} v^{*}}{x_{1}^{*} v}\right)\right. \\
& \left.+F\left(\frac{1+a_{1} x+b_{1} v}{1+a_{1} x+b_{1} v^{*}}\right)+F\left(\frac{x_{1}^{*} x_{\tau_{1}} v_{\tau_{1}}}{x_{1} x^{*} v^{*}\left(1+a_{1} x^{*}+b_{1} v_{\tau_{1}}+b_{1} v_{\tau_{1}}\right)}\right)\right] \\
& -\delta y_{1}^{*} e^{m_{2} \tau_{2}} \gamma\left[F\left(\frac{y^{*}}{y} \frac{1+a_{2} y+b_{2} v^{*}}{1+a_{2} y^{*}+b_{2} v^{*}}\right)+F\left(\frac{y_{1} v^{*}}{y_{1}^{*} v}\right)\right. \\
& \left.+F\left(\frac{1+a_{2} y+b_{2} v}{1+a_{2} y+b_{2} v^{*}}\right)+F\left(\frac{y_{1}^{*} y_{\tau_{2}} v_{\tau_{2}}\left(1+a_{2} y^{*}+b_{2} v^{*}\right)}{y_{1} y^{*} v^{*}\left(1+a_{2} y_{\tau_{2}}+b_{2} v_{\tau_{2}}\right)}\right)\right] .
\end{aligned}
$$

It is easy to see that if $x^{*}, x_{1}^{*}, y^{*}, y_{1}^{*}, v^{*}>0$, then $\frac{d W_{2}}{d t} \leq 0$ for all $\left(x, x_{1}, y, y_{1}, v\right)$ $>0$ where the equality holds if and only if $\left(x, x_{1}, y, y_{1}, v\right)$ take the steady state value $\left(x^{*}, x_{1}^{*}, y^{*}, y_{1}^{*}, v^{*}\right)$. LaSalle's Invariance Principle implies global stability of $E_{1}$.

\section{Conclusion}

In this paper, we have studied the global stability of two HIV infection models with intracellular delays accounting for the times between viral entry into the target cells, the $\mathrm{CD} 4^{+} \mathrm{T}$ and macrophages, and the production of new virus particles. The first model takes into account the saturation infection rate. In the second model the infection rate is given by Beddington-DeAngelis functional response. The global stability of the uninfected and infected steady states have been established by using suitable Lyapunov functionals and LaSalle Invariant Principle. We have proven that, if the basic reproduction number $R_{0}$ is less than unity, then the uninfected steady state is GAS and if the infected steady state exists then it is GAS for all time delays.

\section{References}

[1] D. S. Callaway and A. S. Perelson, HIV-1 infection and low steady state viral loads, Bull. Math. Biol. 64 (2002), 29-64. 
[2] R. V. Culshaw and S. Ruan, A delay-differential equation model of HIV infection of $\mathrm{CD4}_{4}^{+}$T-cells, Math. Biosci. 165 (2000), 27-39.

[3] A. M. Elaiw, Global properties of a class of HIV models, Nonlinear Anal. Real World Appl. 11 (2010), no. 4, 2253-2263.

[4] A. M. Elaiw, I. A. Hassanien, and S. A. Azoz, Global properties of a class of HIV models with Beddington-DeAngelis functional response, (submitted).

[5] A. M. Elaiw and X. Xia, HIV dynamics: Analysis and robust multirate MPC-based treatment schedules, J. Math. Anal. Appl. 356 (2009), no. 1, 285-301.

[6] J. K. Hale and S. Verduyn Lunel, Introduction to Functional Differential Equations, Springer, New York, 1993

[7] A. V. M. Herz, S. Bonhoeffer, R. M. Anderson, R. M. May, and M. A. Nowak, Viral dynamics in vivo: Limitations on estimates of intracellular delay and virus decay, Proc. Natl. Acad. Sci. USA 90 (1996), 7247-7251.

[8] Z. Hu, X. Liu, H. Wang, and W. Ma, Analysis of the dynamics of a delayed HIV pathogenesis model, J. Comput. Appl. Math. 234 (2010), no. 2, 461-475.

[9] G. Huang, W. Ma, and Y. Takeuchi, Global analysis for delay virus dynamics model with Beddington-DeAngelis functional response, Appl. Math. Lett. 24 (2011), no. 7, 1199-1203.

[10] G. Huang, Y. Takeuchi, and W. Ma, Lyapunov functionals for delay differential equations model of viral infection, SIAM J. Appl. Math. 70 (2010), no. 7, 2693-2708.

[11] D. Li and W. Ma, Asymptotic properties of a HIV-1 infection model with time delay, J. Math. Anal. Appl. 335 (2007), no. 1, 683-691.

[12] M. Y. Li and H. Shu, Global dynamics of an in-host viral model with intracellular delay, Bull. Math. Biol. 72 (2010), no. 6, 1492-1505.

[13] J. Mittler, B. Sulzer, A. Neumann, and A. Perelson, Influence of delayed virus production on viral dynamics in HIV-1 infected patients, Math. Biosci. 152 (1998), 143-163.

[14] Y. Nakata, Global dynamics of a viral infection model with a latent period and Beddington-DeAngelis functional response, Nonlinear Anal. 74 (2011), no. 9, 2929-2940.

[15] _ Global dynamics of a cell mediated immunity in viral infection models with distributed delays, J. Math. Anal. Appl. 375 (2011), 14-27.

[16] P. W. Nelson, J. Murray, and A. Perelson, A model of HIV-1 pathogenesis that includes an intracellular delay, Math. Biosci. 163 (2000), no. 2, 201-215.

[17] P. W. Nelson and A. S. Perelson, Mathematical analysis of delay differential equation models of HIV-1 infection, Math. Biosci. 179 (2002), no. 1, 73-94.

[18] M. A. Nowak and R. M. May, Virus Ddynamics: Mathematical Principles of Immunology and Virology, Oxford Uni., Oxford, 2000.

[19] A. S. Perelson, P. Essunger, Y. Cao, M. Vesanen, A. Hurley, K. Saksela, M. Markowitz, and D. D. Ho, Decay characteristics of HIV-1-infected compartments during combination therapy, Nature 387 (1997), 188-191.

[20] A. S. Perelson and P. W. Nelson, Mathematical analysis of HIV-1 dynamics in vivo, SIAM Rev. 41 (1999), no. 1, 3-44.

[21] X. Shi, X. Zhou, and X. Song, Dynamical behavior of a delay virus dynamics model with CTL immune response, Nonlinear Anal. Real World Appl. 11 (2010), no. 3, 1795-1809.

[22] X. Song, S. Wang, and J. Dong, Stability properties and Hopf bifurcation of a delayed viral infection model with lytic immune response, J. Math. Anal. Appl. 373 (2011), no. 2, 345-355.

[23] X. Song, X. Zhou, and X. Zhao, Properties of stability and Hopf bifurcation for a HIV infection model with time delay, Appl. Math. Model. 34 (2010), no. 6, 1511-1523.

[24] X. Wang, Y. Tao, and X. Song, A delayed HIV-1 infection model with BeddingtonDeAngelis functional response, Nonlinear Dynam. 62 (2010), no. 1-2, 67-72.

[25] Y. Wang, Y. Zhou, J. Heffernan, and J. Wu, Oscillatory viral dynamics in a delayed HIV pathogenesis model, Math. Biosci. 219 (2009), no. 2, 104-112. 
[26] Q. Xie, D. Huang, S. Zhang, and J. Cao, Analysis of a viral infection model with delayed immune response, Appl. Math. Model. 34 (2010), no. 9, 2388-2395.

[27] R. Xu, Global stability of an HIV-1 infection model with saturation infection and intracellular delay, J. Math. Anal. Appl. 375 (2011), no. 1, 75-81.

[28] H. Zhu, Impact of delays in cell infection and virus production on HIV-1 dynamics, Math. Medic. Biol. 25 (2008), 99-112.

Ahmed Elaiw

Department of Mathematics

FACULTy OF SCIENCE

King Abdulaziz University

P.O. Box 80203, JedDAh 21589, SAudi Arabia

AND

Department of Mathematics

FACUlty of SCIENCE

Al-AzHAR UNIVERSITY

Assiut 71511, EGYPT

E-mail address: a_m_elaiw@yahoo.com

ISMAIL HASSANIEN

Department of Mathematics

FACUlty of Science

Assiut University

Assiut 71516, EGYPT

E-mail address: s_hassanienby@yahoo.com

SHIMAa AzOz

Department of Mathematics

Faculty of Science

Assiut University

Assiut 71516, EGYPT

E-mail address: shimaa_azoz@yahoo.com 